\title{
Noise reduction in dual-thickness laser-based T-ray material characterization
}

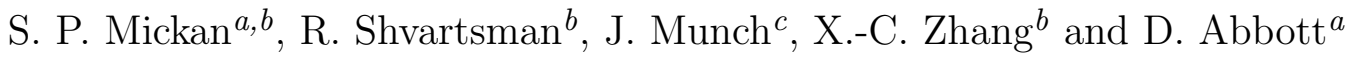 \\ ${ }^{a}$ Centre for Biomedical Engineering (CBME) and Department of Electrical \& Electronic \\ Engineering, The University of Adelaide 5005, Australia. \\ ${ }^{b}$ Center for Terahertz Research and Department of Physics, Applied Physics \& Astronomy, \\ Rensselaer Polytechnic Institute, Troy NY 12180, USA. \\ ${ }^{c}$ Department of Physics \& Mathematical Physics, The University of Adelaide 5005, Australia.
}

\begin{abstract}
Pulsed THz (T-ray) spectroscopy is an increasingly common tool for studying materials from physics to biology. Liquid transmission T-ray studies are valuable for understanding solvation dynamics of salts, exploring longrange structure in mixtures and probing biomolecules in suspension. In this paper the uncertainty in parameter estimation of liquid samples is shown to be dependent on the thickness change of the sample, and on the noise of the T-ray spectrometer. For many important liquids, such as water, with high THz absorption, we show that measurement uncertainty can be greatly decreased using a dynamically-modulated liquid sample, using differential T-ray time-domain spectroscopy (DTDS). Preliminary experiments with a novel sample holder, based on a loud speaker, support these calculations. Amplitude and mean detection is used to simultaneously measure two waveforms in DTDS, potentially decreasing the noise by $10^{2}$.
\end{abstract}

Keywords: T-ray, differential $\mathrm{THz}$ time-domain spectroscopy

\section{INTRODUCTION}

The T-ray radiation band lies at the boundary between electronics and photonics, approximately 0.1 to $10 \mathrm{THz}$. T-rays overlap with high-frequency mm-waves and the long wavelengths of the far-infrared. T-rays have a diffraction-limited spatial resolution limited by wavelength, $\lambda$, to approximately $0.3 \mathrm{~mm}$ at $1 \mathrm{THz}$, which is better than millimetre (mm)-waves, and T-ray Rayleigh scattering is less than for IR light due to its $\lambda^{-4}$ dependence. The approximate bandwidth of typical pulsed T-ray spectrometers, as used for experiments in this paper, spans from $300 \mathrm{GHz}$ to $3 \mathrm{THz}^{1-4}$

The accuracy of estimating the T-ray frequency parameters of fluid samples in dual-thickness T-ray spectroscopy is limited by fluctuations in the T-ray pulses, caused by laser fluctuations, and by the accuracy of the thickness measurements. ${ }^{5,6}$

The measurement of thickness change $d$ can be extremely accurate, better than 1:10 ${ }^{4}$, using closed-loop feedback control of the mechanism used for changing the sample thickness.

Noise in the T-ray beam is caused primarily by laser fluctuations, which are known to have a $1 / f$ characteristic, that is, the fluctuation amplitude is greater at lower frequencies. A measured graph of T-ray spectrometer noise is shown in Fig. 1, where the $1 / f$ trend is visible. Normally the two measurements required for material characterization are measured over a period of minutes. The length of time required depends on the signalto-noise ratio (SNR) of the system. By taking the reference and sample measurements, required in T-ray spectroscopy, at a higher speed, the fluctuation in laser power between measurements is reduced. Rapid sample modulation, dubbed T-ray Differential Time-Domain Spectroscopy (DTDS), has previously been used on thin films ${ }^{7-9}$ biosensor slides, ${ }^{10,11}$ but not on liquid or gas samples.

S. P. Mickan Email: spmickan@eleceng.adelaide.edu.au; R. Shvartsman Email: shvarr@rpi.edu; J. Munch Email: jmunch@physics.adelaide.edu.au; X.-C. Zhang Email: zhangxz@rpi.edu; D. Abbott Email: dabbott@eleceng.adelaide.edu.au. 
Liquid spectroscopy is best carried out with two thickness measurements, using a thick sample and a thin sample of the liquid. Dual-thickness measurements can be made at higher modulation frequencies by rapidly swapping between the thick and thin samples. This paper describes the theory of the novel technique of liquid DTDS. Experiments with a prototype sample modulation system based on an audio loud speaker are used to demonstrate the feasibility of liquid DTDS.

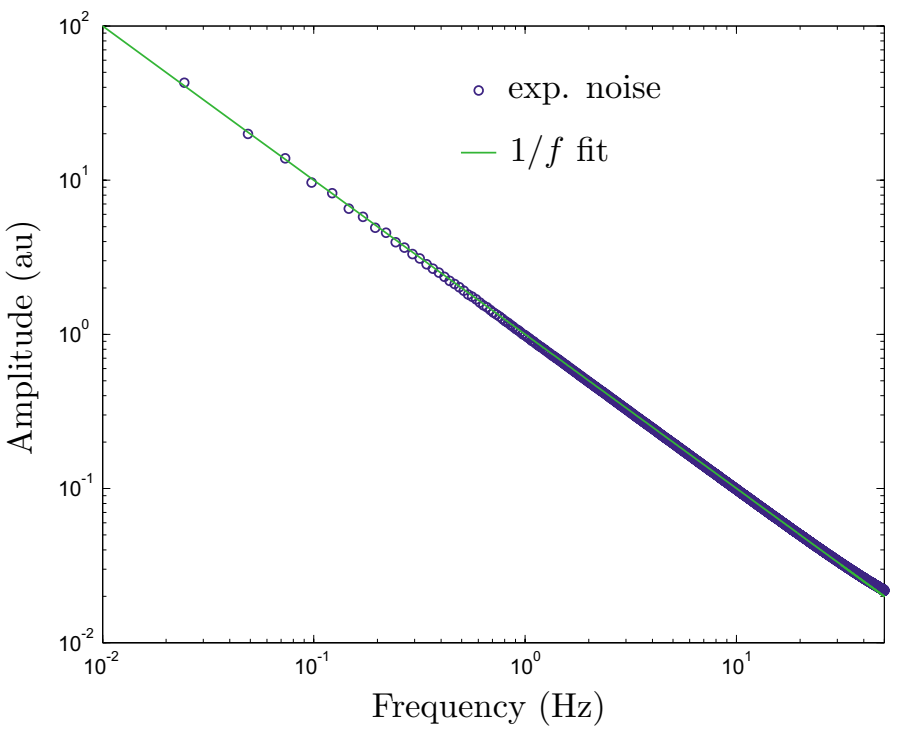

Figure 1. Experimental T-ray noise spectrum. The $1 / f$ trend of laser-based T-ray noise is visible in this experimental spectrum. The spectrum was calculated from a set of time-domain measurements of the peak of the T-ray pulse. The 8,192 time-domain samples were taken with a time spacing of approximately $5 \mathrm{~ms}$, giving a sample time of $41 \mathrm{~s}$ and a measurement frequency range of approximately 0.024 to $100 \mathrm{~Hz}$. Liquid DTDS would decrease the drift time from approximately 5 mins to $0.05 \mathrm{~s}(0.003 \mathrm{~Hz}$ to $50 \mathrm{~Hz})$, theoretically decreasing the noise caused by laser fluctuations by up to 4 orders of magnitude.

\subsection{Previous studies}

T-ray spectroscopy of fluids has been used to study liquid dynamics, solvation processes and biological systems. ${ }^{12-24}$ T-ray fluid parameter studies have been carried out with a variety of spectrometer configurations and parameter estimation procedures. The experiments in this paper use a dual-sample geometry, where the sample and reference waveforms are measured through thick and thin versions of the sample respectively. Dualthickness geometry systems have been used by Ref. 17 and Ref. 6 .

The typical accuracy of determined parameters is to 2 decimal places, or worse than $0.1 \% .{ }^{13,20,24-26}$ The work in this paper attempts to improve the accuracy of these measurements by 2 orders of magnitude.

\subsection{Objective}

This paper proposes to use double-modulated DTDS to reduce the noise in fluid parameter estimation. In a typical T-ray transmission spectrometer with an SNR of 100:1, each time-domain measurement takes approximately 5 mins, and noise is dominated by laser fluctuations $(1 / f)$. Using a DTDS technique and simultaneous dual waveform acquisition (amplitude and mean detection), the time between measuring the sample and reference waveforms can be reduced to a time determined by the speed of the modulator. Therefore only one delay scan is required to sample both T-ray waveforms, and the time delay between each sample and reference measurement is the inverse of the modulation frequency. For a $10-\mathrm{Hz}$ modulation speed, the increase in effective modulation frequency is 3000 times, and the corresponding decrease in the amplitude of $1 / f$ noise is 3000 times.

\section{THEORETICAL NOISE REDUCTION}

For liquid spectroscopy using a dual sample geometry, the accuracy of parameter estimation depends on fluctuation of the T-ray spectrometer and the accuracy of the thickness change. This section quantifies the benefits of DTDS for characterising two classes of liquids: those with a low T-ray absorption, for example non-polar solvents, and those with a high T-ray absorption, for example water. The aim is to measure material parameters with an accuracy better then $10^{-4}$. 


\subsection{Dual-sample theory}

The T-ray pulse is modeled as a spectrum of Fourier components, propagating as a plane wave through the sample. Figure 2.1 shows the propagation of radiation through the sample, of complex refractive index $\tilde{n_{2}}$, and across two interfaces between the sample and the surrounding medium (air), $\tilde{n_{1}} \approx 1.0$. The simple equations used for estimating the material parameters are derived from the equations for Fresnel transmission and reflection at interfaces.

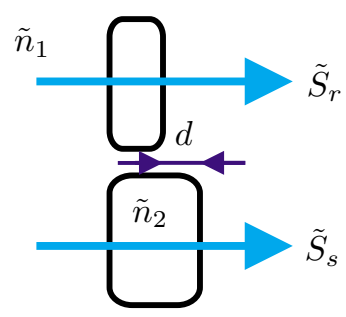

Figure 2. Dual-thickness parameter estimation geometry. Waveforms $y_{s}$ and $y_{r}$ are measured having respectively passed through the thick (sample) and thin (reference) samples. The spectral components of the waveforms are determined by FFT, $\tilde{S}_{s}=$ $\mathcal{F} \mathcal{T}\left(y_{s}\right)$ and $\tilde{S}_{r}=\mathcal{F} \mathcal{T}\left(y_{r}\right)$. The difference in thickness between the two measurements is denoted $d$.

A thick sample is a sample that causes sufficient delay so that the transmitted pulse can be measured without any overlap with the first Fabry-Pérot (FP) reflection. The exact requirements for the delay will depend on the desired total scan length. Note that the substrate material both before and after the sample must also be sufficiently thick to avoid any FP reflections in the measured pulses. Thin substrates are discussed below with thin samples.

For a thick sample, the experimentally-measured spectral components of the reference and sample pulses can be modeled by ${ }^{4,27,28}$

$$
\tilde{S}_{r}(\omega)=A(\omega) \cdot t_{12} \cdot p_{d_{\mathrm{thin}}} \cdot t_{21}, \quad \tilde{S}_{s}(\omega)=A(\omega) \cdot t_{12} \cdot p_{d_{\mathrm{thick}}} \cdot t_{21}
$$

where $A(\omega)$ is the product of all other system responses that remain constant between the sample and reference measurements. For an interface between two media of refractive indicies $\tilde{n}_{a}$ and $\tilde{n}_{b}$, oriented normal to the radiation path, the plane wave transmission $(t)$ and reflection $(r)$ equations simplify to ${ }^{27,29}$ :

$$
\begin{aligned}
t_{a b}(\omega) & =\frac{2 \tilde{n}_{a}}{\left(\tilde{n}_{a}+\tilde{n}_{b}\right)}, \text { and } \\
r_{a b}(\omega) & =\frac{\tilde{n}_{a}-\tilde{n}_{b}}{\tilde{n}_{a}+\tilde{n}_{b}} .
\end{aligned}
$$

Radiation propagating a distance $d_{a}$ through a linear medium, $\tilde{n}_{a}$, is delayed and attenuated according to the factor

$$
p(\omega)=e^{-j \tilde{n}_{a} \omega d / c_{0}}
$$

where $c_{0}$ is the speed of light in a vacuum.

The ratio of the transmission spectra can thus be entirely determined in terms of refractive indicies and the sample thickness, ${ }^{6,27,29}$

$$
\tilde{T}=\tilde{S}_{\text {sample }} / \tilde{S}_{\text {reference }}=\frac{e^{-j \tilde{n}_{2} \omega d_{\text {thick }} / c_{0}}}{e^{-j \tilde{n}_{2} \omega d_{\text {thin }} / c_{0}} e^{-j \tilde{n}_{1} \omega\left(d_{\text {thick }}-d_{\text {thin }}\right) / c_{0}}},
$$

where $\tilde{n}_{1}$ is the material that replaces the space taken up by the extra thickness of the thick sample, typically air.

Equation (4) simplifies to

$$
\tilde{T}=\exp \left(-j \omega / c_{0} d\left(\tilde{n}_{2}-1\right)\right)
$$


where $d=d_{\text {thick }}-d_{\text {thin }}$ and $\tilde{n}_{1}=1$. Since $\tilde{T}$ is complex, $\tilde{T}=\rho e^{j \phi}$, and $\tilde{n}=n-j \kappa$,

$$
\rho=\exp \left(-\omega d \kappa / c_{0}\right), \quad \phi=\omega d\left(n_{2}-1\right) / c_{0} .
$$

The material parameters can then be extracted by rearranging the above equations, ${ }^{30}$

$$
n=\frac{-\phi c_{0}}{\omega d}+1, \quad \kappa=-\ln (\rho) \frac{c_{0}}{\omega d} .
$$

These calculations provide a value for the T-ray refractive index and extinction of the mixture. The values of $\tilde{S}_{s}$ and $\tilde{S}_{r}$ were measured by taking the Fourier transform of the transmitted time-domain T-ray pulses, $y_{s}$ and $y_{r}$ respectively. The accuracy of Eq. (7) depends only on the accuracy of $d$ and on the noise of the T-ray spectrometer signal, $\partial \rho \& \partial \phi$.

\subsection{Uncertainty}

The influence of errors arising from the thickness measurement, $\partial d$, and from T-ray spectrometer noise, $\partial \rho \&$ $\partial \phi$, can be estimated using the partial derivatives

$$
\partial n=\frac{\phi c_{0}}{\omega} \frac{1}{d^{2}} \partial d, \quad \partial \kappa=\frac{\ln (\rho) c_{0}}{\omega} \frac{1}{d^{2}} \partial d
$$

and

$$
\partial n(\omega)=-\frac{c_{0}}{\omega d} \partial \phi(\omega), \quad \partial \kappa(\omega)=-\frac{c_{0}}{\omega d} \frac{(\omega)}{\rho(\omega)} .
$$

The T-ray noise comes from three main sources: (i) the T-ray emitter, (ii) T-ray detector and (iii) the probe beam. The noise in the emitter dominates and is multiplicative, that is, proportional to the signal strength. The noise in the probe beam is additive, that is, independent of signal strength, and only dominates when the signal strength is low. For experiments dominated by multiplicative noise, that is, noise due to fluctuations in the T-ray signal (pump beam) rather than noise in the detection system (probe beam), ${ }^{31}$ the phase error is approximately equal in both the sample and reference spectra,

$$
\partial \phi_{S} \approx \partial \phi_{R}
$$

From $\phi=\phi_{S}-\phi_{R}$ and Eq. (2.2), $\partial \phi \approx 2 \partial \phi_{R}$.

For uncorrelated amplitude noise in both the sample and reference, the combined effect of noise in both sample and reference can be estimated from

$$
\left(\frac{\partial \rho}{\rho}\right)^{2}=\left(\frac{\partial \rho_{R}}{\rho_{R}}\right)^{2}+\left(\frac{\partial \rho_{S}}{\rho_{S}}\right)^{2} .
$$

For multiplicative noise, the relative amplitude error is similarly approximately equal in both the sample and reference spectra, giving

$$
\begin{aligned}
\frac{\partial \rho}{\rho} & \approx \sqrt{2} \frac{\partial \rho_{R}}{\rho_{R}} \\
\partial \rho & =\frac{\sqrt{2} \exp \left(\frac{-\omega d k}{c_{0}}\right)}{\rho_{R}} \partial \rho_{R} .
\end{aligned}
$$

Using Eq. (6), and the relations for uncorrelated noise sources,

$$
\partial n=\sqrt{\left(\partial n_{\phi}\right)^{2}+\left(\partial n_{d}\right)^{2}}, \quad \partial k=\sqrt{\left(\partial k_{\rho}\right)^{2}+\left(\partial k_{d}\right)^{2}}
$$


expressions for the uncertainty in measurements due to phase, amplitude and thickness uncertainties are obtained:

$$
\begin{aligned}
\partial n & =\frac{n-1}{d} \sqrt{\left(\frac{2 c_{0} \partial \phi_{R}}{\omega(n-1)}\right)^{2}+(\partial d)^{2}}, \\
\partial k & =\frac{k}{d} \sqrt{\left(\frac{\sqrt{2} c_{0}}{\omega k} \cdot \frac{\partial \rho_{R}}{\rho_{R}}\right)^{2}+(\partial d)^{2}} .
\end{aligned}
$$

The uncertainty in the estimation of refractive index and extinction coefficient can be predicted from the inherent noise in the T-ray spectrometer, $T_{R}$, and the accuracy of the thickness modulator measuring $d$.

To study the influence of these uncertainties, we look at four cases: (i) $\partial d$ dominant in a low- $k$ sample (for example, a non-polar solvent); (ii) $\partial \rho$ and $\partial \phi$ dominant in a very thick low- $k$ sample; (iii) $\partial d$ dominant in a high- $k$ sample (for example, water); and (iv) $\partial \rho$ and $\partial \phi$ dominant in a high- $k$ sample.

\subsection{1. $\partial d$ dominant in low- $\kappa$}

For noise dominated by errors in $d$, Eqs. (12) and (13) can be simplified by ignoring the first term in the square root, giving

$$
\partial n=(n-1) \frac{\partial d}{d}, \quad \partial k=k \frac{\partial d}{d} .
$$

Equation (14) indicates that noise can be reduced by using a large thickness change $d$ compared to the thickness measurement error $\partial d$. $\partial d$ is limited by the actuator resolution. $\frac{\partial d}{d}<10^{-4}$ can be achieved by using, for example, a 5 -mm distance change between thicknesses and $0.4-\mu \mathrm{m}$ stage resolution, or a $30-\mu \mathrm{m}$ piezoelectric stage with 1-nm resolution. An accuracy of $10^{-4}$ would constitute an improvement of 100 times over most Tray liquid characterization results. Such high-accuracy mechanical modulators are commercially available. The dominant source of noise is typically spectrometer noise, $\partial \rho \& \partial \phi$.

\subsection{2. $\partial \rho$ and $\partial \phi$ dominant in low- $\kappa$}

For $T$-dominated errors, Eqs. (12) and (13) can be simplified by ignoring the second term in the square root, giving

$$
\partial n=\frac{2 c_{0} \partial \phi_{R}}{\omega d}, \quad \partial k=\frac{\sqrt{2} c_{0}}{\omega d} \cdot \frac{\partial \rho_{R}}{\rho_{R}} .
$$

These equations can be understood by substituting approximate values from a typical T-ray spectrometer measurement. For example, $\omega=6.3 \times 10^{12}$ at a frequency of $1 \mathrm{THz}$, and $\rho_{R}=0.655, \partial \rho_{R}=0.015$ and $\partial \phi_{R}=0.025 \mathrm{rad}$ for a scan of the organic solvent 1,4-dioxane, using the liquid T-ray spectrometer from Sec. 3. In this measurement, the LIA time constant was $100 \mathrm{~ms}$, giving a scan duration of approximately 5 mins. Using these numbers,

$$
\partial n \approx \frac{5 \cdot 10^{-6}}{d}, \quad \partial k \approx \frac{3 \cdot 10^{-6}}{d} .
$$

From Eq. (15) it can be seen that an accuracy of $10^{-4}$ is achievable at this noise level (100-ms LIA time constant and $5 \mathrm{~min}$ scan) by using an approximate thickness change of $d>10 \mathrm{~mm}$. This large thickness chance is possible with large volumes of liquid with a low T-ray absorption. If, however, the T-ray absorption of the liquid is not very low, a 50-mm-thick sample will attenuate the T-rays too greatly to be detected. For high- $\kappa$ samples, the initial noise in the T-ray signal must be reduced to compensate for the necessity of a small thickness change.

\subsection{3. $\partial d$ dominant in high- $\kappa$}

For high- $\kappa$ samples, the uncertainty caused by $\partial d$ is governed by the same equations as for low- $\kappa$ samples, $(2.2 .2)$. For a highly-absorbing liquid, however, it is not possible to use a 5-mm distance change. The sample modulation must be less than $100 \mu \mathrm{m}$ and be measured with a very high accuracy, using for example a piezoelectric translator. 


\subsection{4. $\partial \rho$ and $\partial \phi$ dominant in high- $\kappa$}

For high- $\kappa$ samples, $\tilde{S}_{S}$, is greatly attenuated, and $\partial \rho$ is dominated by additive noise.

Thus

$$
\begin{aligned}
\partial k & =\frac{\sqrt{2} c_{0}}{\omega d} \cdot \frac{\partial \rho_{S}}{\exp \left(\frac{-\omega d k}{c_{0}}\right)}, \\
& \approx \frac{1.3 \cdot 10^{-4}}{d} \partial \rho_{S} \frac{1}{\rho} .
\end{aligned}
$$

The error is proportional to $1 / \rho$. We can compare the influence that increasing the modulation thickness, $d$, has on the accuracy of our estimations when using two different liquids, dioxane $\left(\kappa_{\text {dioxane }}=0.013\right)$ and water $\left(\kappa_{\text {water }}=0.478\right)$. The graph in Fig. 3 shows that the error in $k$ measurements of a high- $k$ liquid, water, increases with sample thickness. For a low- $k$ liquid, dioxane, the error decreases with increasing thickness.

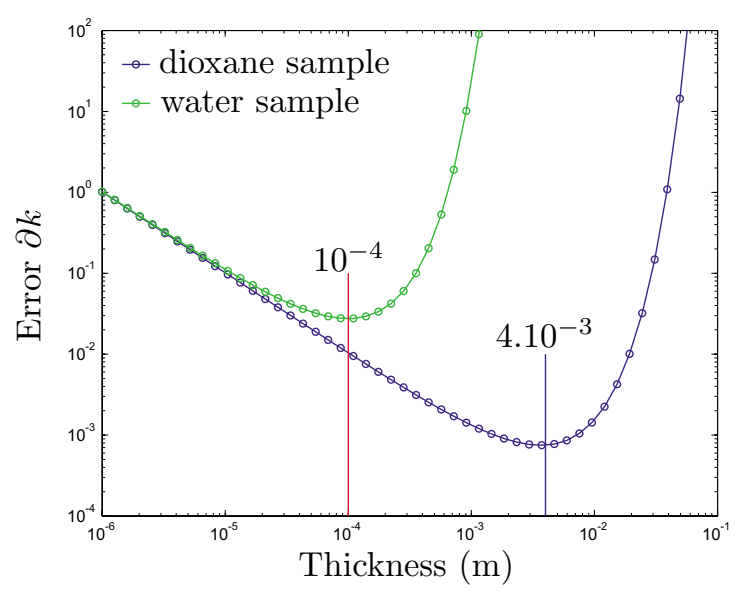

Figure 3. $\partial k$ for changing liquid thickness. The two liquids in the graph are dioxane, $\kappa=0.013$, and water, $\kappa$ $=0.478$. For the typical error in a T-ray spectrometer, for example $\partial \rho_{s}=0.015$, the error in the estimation of the $k$ parameter depends on the thickness change of the sample. The error decreases for thicker samples, from Eq. (16), but for highly-absorbing liquids, such as water, the increased thickness masks the T-ray signal. These plots show the approximate thickness change desirable for minimum error. For dioxane, a thicker sample is desirable, approximately 4 mm. For water, the thickness change is much less, approximately $100 \mu \mathrm{m}$.

Figure 3 shows that a thickness change of even $1 \mathrm{~mm}$ generates a very high error in a high- $\kappa$ liquid, thus limiting experiments to sub-mm modulation.

\subsubsection{Conclusion}

From the foregoing analysis of uncertainty, we see the best modulation system for liquid spectroscopy depends on the properties of the liquid. For a low- $\kappa$ liquid, a high accuracy can be achieved by using a very large thickness change up to $d=10 \mathrm{~mm}$. For a high- $\kappa$ liquid, only a small thickness change is possible, and the errors must be reduced in two ways: (i) the thickness change must be accurate, for example, by using a piezoelectric translator, and (ii) the inherent noise in the T-ray signal must be reduced, by using for example high-frequency sample modulation in double-modulated DTDS.

For dioxane measurements, an accuracy of $\sim 10^{-4}$ is desirable. From Eq. (15), a thickness change of $\sim 10 \mathrm{~mm}$ is required, with a resolution of $1 \mu \mathrm{m}$. Since dioxane has low absorption, this is possible.

For water measurements, a thickness of $1 \mathrm{~cm}$ is unusable since the T-ray absorption is too high. A thickness change of around $100 \mu \mathrm{m}$ and a resolution of $10 \mathrm{~nm}$ is required, using for example a piezoelectric actuator. 


\section{EXPERIMENT}

This section describes an initial experimental verification of the accuracy of liquid DTDS and discusses its limitations.

The experimental implementation of liquid DTDS involves three main elements: (i) a standard T-ray transmission spectrometer, (ii) a sample holder for modulating the liquid sample at a modulation frequency $f_{2}$, and (iii) electronic signal processing to extract the sample and reference waveforms, $y_{s}$ and $y_{r}$. The spectrometer used in these experiments is the same system used in standard liquid transmission spectroscopy, ${ }^{5,6}$ and is shown schematically in Fig. 4.

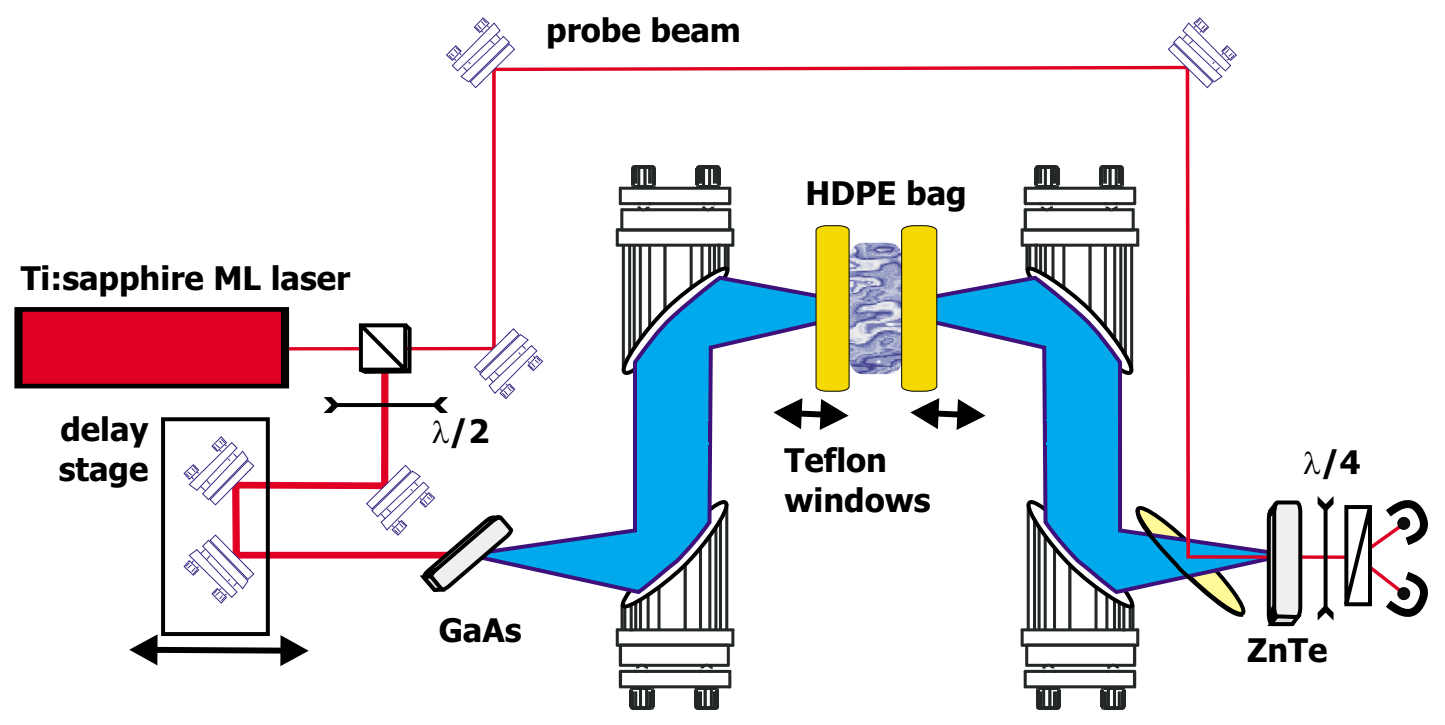

Figure 4. Liquid DTDS spectrometer schematic. This schematic depicts a standard T-ray spectrometer configured for characterising a liquid sample. In liquid DTDS the thickness of the sample is modulated dynamically at a frequency $f_{2}$, and the electronic demodulation is similar to thin film double-modulation DTDS. ${ }^{8,32}$ The Ti:sapphire mode-locked (ML) laser generates a train of $100-\mathrm{fs}$ pulses at $82 \mathrm{MHz}$. Each pulse is split into a pump beam and a probe beam. The pump beam generates T-ray pulses when incident at Brewster's Angle on the GaAs wafer, positioned at the focal point of a gold-coated parabolic reflector. Parabolic reflectors collimate the T-rays, focus it into the sample holder, then collect the transmitted radiation and direct it into a $\langle 110\rangle \mathrm{ZnTe}$ T-ray detector. The probe beam detects the T-ray electric field in the $\mathrm{ZnTe}$, and is in turn recorded with crossed polarisers and balanced photodetectors. The thickness of the sample is modulated with two Teflon windows, one of which was mounted on manual translation stage. The mixture was held in a sealed, thin-walled, high-density polyethylene (HDPE) bag, which does not react with or dissolve in dioxane.

The sample holder needs to provide a rapid transition between a thick and thin sample, corresponding to $y_{s}$ and $y_{r}$ in dual-thickness spectroscopy. In the prototype system this was implemented using one large fixed Teflon window and one moving window mounted on an audio speaker, as shown in Fig. 5. The speaker is driven by a square wave to switch between the thick and thin positions. The signal processing consists of two parts: the standard DTDS double de-modulation ${ }^{8,32}$ to extract the difference waveform $y_{d}=y_{s}-y_{r}$, and a second part to measure the mean waveform $y_{m}=y_{s}+y_{r}$. Amplitude and mean detection is described in Sec. 3.1 below.

The two Teflon windows, held perpendicular to the T-ray beam axis, are thicker than 10 mm to avoid FabryPérot reflections in the recorded waveform. Teflon is a good material for T-ray windows because of its low refractive index and low absorption. The actual liquid samples are held in an high-density polyethylene bag (HDPE), a material that does not dissolve in the solvent. HDPE has a low T-ray refractive index and low T-ray absorption. With an average wall thickness less than $0.1-\mathrm{mm}$ thick, less than the majority of T-ray wavelengths, and a very low refractive index mismatch between Teflon and HDPE, etalon effects from the plastic bag walls are negligible. Experimentally, no multi-reflections are observed in the measured pulses. For a low mismatch between $\tilde{n}_{1}, \tilde{n}_{2}$ and $\tilde{n}_{3}$, and a thin bag wall, the influence of etalon effects is slight. 


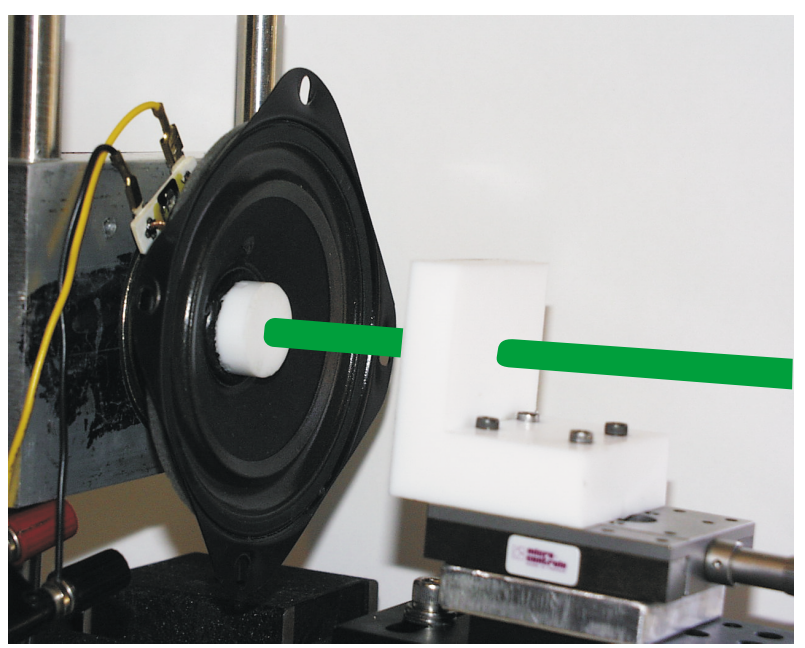

Figure 5. Liquid DTDS sample holder. Photograph of the prototype liquid sample holder. The speaker is an RCA 4 "-diameter mid-range speaker, with $8-\Omega$ DC coil resistance. The speaker is driven by a square wave of frequency $f_{2}$ from an SRS signal generator (DS340), and travels axially along the T-ray beam propagation direction.

\subsection{Amplitude and mean detection}

Amplitude and mean detection is an electronic technique that enables the simultaneous measurement of both the sample and reference waveforms when a sample is modulated in the T-ray beam. Amplitude and mean detection can be used in any DTDS experiment and is implemented as part of the signal detection electronics. In amplitude and mean detection, instead of discarding $50 \%$ of the $y_{d}$ waveform as in double-modulated DTDS, ${ }^{8,9,32}$ half the signal is used to simultaneously measure $y_{r}$. The advantage of this is that the hardware requires no modification from double-modulated DTDS and that the time between measuring $y_{d}$ and $y_{r}$ is reduced from the order of minutes to the order of milliseconds. For measurements dominated by $1 / f$ noise, a reduction in the scan-to-scan delay time increases the SNR, potentially by several orders of magnitude.

A mean and amplitude detection system can be implemented in stand-alone electronics, using a fast analogto-digital converter $\left(>>f_{1}\right)$ and 2 stages of digital demodulation, synchronised with the movement of the delay stage. The diagram in Fig. 6 shows schematically how the double-modulated signal from the photodiodes is demodulated in 2 stages. The double demodulation process can be understood in the time domain by looking at the square waveform detected by the photodiodes as the sample is modulated differentially. An example detected waveform is shown in Fig. 7, showing how $y_{\mathrm{m}}$ and $y_{\mathrm{a}}$ relate to $y_{s}$ and $y_{r}$ at the output of the first mixer.

It is possible to simultaneously record the sample and reference waveforms in one scan when the sample is being dithered in and out of the T-ray beam, as in DTDS. In order to measure both $y_{s}$ and $y_{r}$ at a modulation frequency of $f_{2}$, two values are found for each delay point, and as the delay stage moves (Fig. 4), two waveforms are plotted, $y_{\mathrm{m}}$ and $y_{\mathrm{a}}$. At each point of the scanning delay line, first the reference signal is measured, then the sample signal. This can be done by having the sample on a dithering mechanism, as in DTDS. The T-ray beam is modulated at an audio frequency $f_{1}$ and the sample is dithered at some low frequency, $f_{2}$. For example, $f_{1}=$ $2 \mathrm{kHz}$ and $f_{2}=10 \mathrm{~Hz}$. In double-modulated DTDS, the $f_{1}$ carrier frequency is demodulated and the amplitude of $f_{2}$ waveform is monitored. As mentioned above, this is in fact the difference between the two waveforms. By using an integrator (low-pass filter), we can simultaneously monitor the mean value of the 10-Hz square wave, which gives (half) the sum of the two waveforms. The mean is the standard output of the first LIA in Fig. 6 . The amplitude is the output of the second LIA used in double-modulated DTDS. Thus $y_{r}$ and $y_{s}$ can be calculated at each point in the same time that it takes to do a normal DTDS scan.

The experiment can be realised with an anti-aliasing filter, a local oscillator referenced to $f_{1}$, a local oscillator referenced to $f_{2}$ and an integrator, as shown in Fig. 6. In the experimental work in this thesis, the system was implemented with three LIAs. The same effect could be achieved digitally with a low-noise preamplifier, a fast analog-to-digital converter and a dedicated processor. The factors of 2 are missing in Fig. 6 because $y_{r}$ and $y_{s}$ 


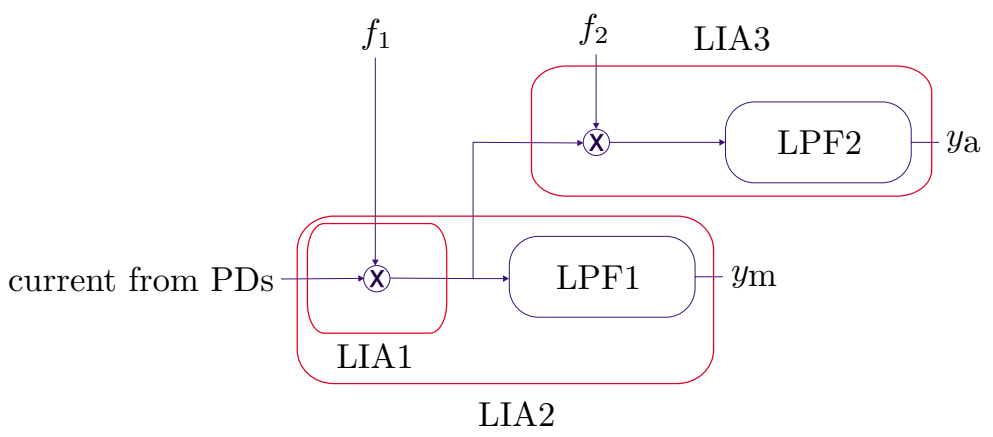

Figure 6. Schematic of mean and amplitude detection. The double-modulated signal from the balanced photodiodes (PDs) is demodulated in two stages. The first demodulation by the higher frequency $f_{1}$ is followed by a narrow band low-pass filter LPF1. The second demodulation by the low frequency $f_{2}$ is again followed by the narrow band LPF2. $y_{r}=y_{\mathrm{m}}-y_{\mathrm{a}}$ and $y_{s}=y_{\mathrm{m}}+y_{\mathrm{a}}$. Experimentally, each of the blocks shown can be implemented with a LIA and the bandwidth of the LPFs are determined by the time constants of the LIAs. LIA1 acts as the $f_{1}$ demodulator and LIA2 acts as the amplitude detector with a long time constant (approximately $100 \mathrm{~ms}$ ), demodulating at $f_{2}$. A third LIA, LIA3, also with a longer time constant $(100 \mathrm{~ms})$ acts as a $f_{1}$ demodulator and detects the mean waveform. No low-pass filtering is required in LIA1, so its bandwidth is set very broad (30- $\mu$ s time constant).

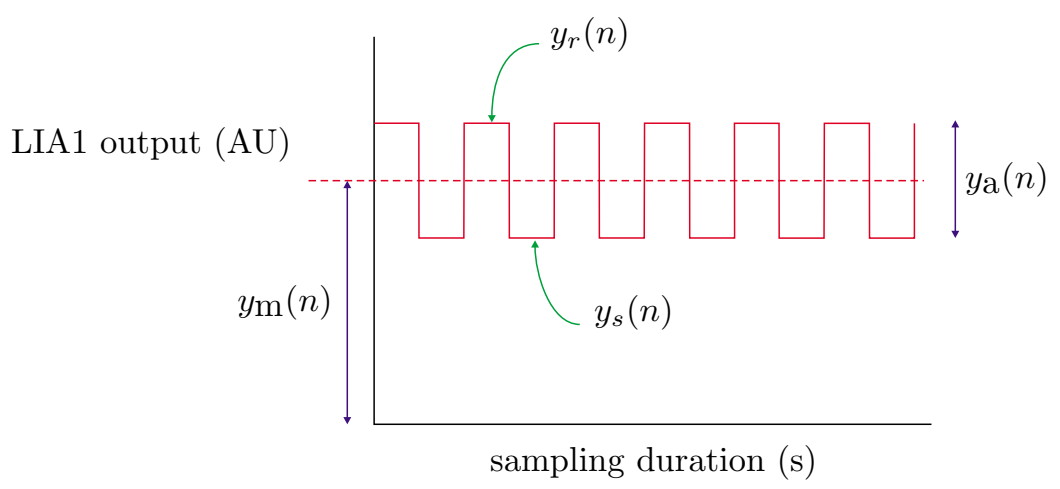

Figure 7. Amplitude and mean detection at one delay point. This figure shows the output of the first demodulator (for example, LIA1) being modulated by the sample modulation (for example, changing the liquid sample thickness). This is the time-domain waveform seen for the $n$th step of the delay stage. The relationship between $y_{\mathrm{m}}, y_{\mathrm{a}}, y_{s}$ and $y_{r}$ can be seen in the diagram. The errors arising from amplitude and mean detection are caused by deviations of the observed signal from an ideal square wave, and this depends on the physical constraints of the system, as discussed in Sec. 2.2. 
are effectively halved by dithering the sample. The trade-off is that although the signal level is halved, but the two waveforms are measured in the same period of time.

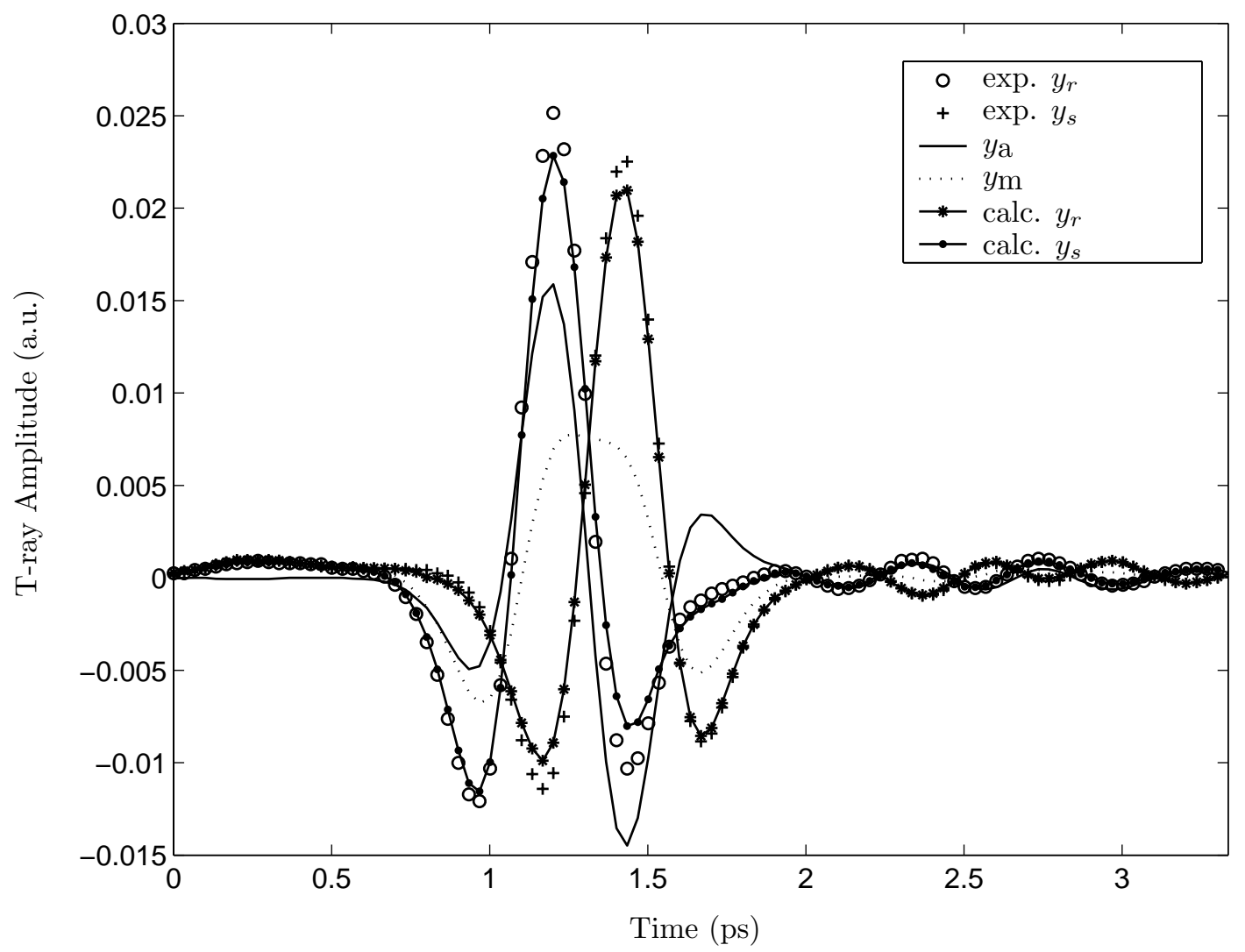

Figure 8. Amplitude and mean detection. Example of single-scan DTDS. The waveforms $y m$ and $y$ a were measured with double-modulated DTDS, using the system shown in Fig. 6. The "calc. $y$ " waveforms were calculated from $y_{r}=$ $y \mathrm{~m}-y_{\mathrm{a}} \& y_{s}=y_{\mathrm{m}}+y_{\mathrm{a}}$. "exp. $y_{r}$ " and "exp. $y_{s}$ " were measured using the exactly the T-ray spectrometer and sample, but without modulating the sample and taking two separate sample and reference scans (normal T-ray TDS). The match between "calc. $y$ " and "exp. $y$ " is an experimental confirmation that amplitude and mean detection provides exactly the same information about a sample as normal T-ray TDS, but with a potentially far lower noise level.

Figure 8 experimentally demonstrates the technique of mean and amplitude detection in the T-ray spectrometer. The mean and amplitude waveforms are taken as shown in Fig. 6, which effectively means the outputs of the 2nd and 3rd LIAs respectively. The accuracy of the technique is confirmed by comparing the calculated waveforms, from $y_{r}=y_{\mathrm{m}}-y_{\mathrm{a}} \& y_{s}=y_{\mathrm{m}}+y_{\mathrm{a}}$ using amplitude and mean data, to two waveforms measured using normal T-ray TDS, "exp. $y_{r}$ " and "exp. $y_{s}$ ". The slight difference between "calc. $y_{r}$ " and "exp. $y_{r}$ " in Fig. 8 is due to sample being modulated by an imperfect square wave. This is a mechanical error introduced by the prototype mechanical sample modulation arm. ${ }^{8}$

\section{RESULTS}

The results for a preliminary demonstration of liquid DTDS show that liquid spectroscopy is functional, and show the technique's limitations. The difference waveform $y_{d}$ is shown experimentally to be equal to $y_{r}-y_{s}$, as required for the DTDS analysis. The accuracy of the measurements, which is critical to accurate sample characterization, is shown to depend on the static linearity of the actuator (audio speaker), the dynamic nonlinearities introduced at higher modulation frequencies and larger modulation amplitudes. The goal in a liquid DTDS system is to have as large a movement as possible at as high a frequency as possible. A large movement (thickness change $d$ ) increases the accuracy of the parameter estimation (Sec. 2). A higher modulation frequency 
allows for less $1 / f$ fluctuation. The best possible modulation amplitude and frequency depends on mechanical limitations of the sample holder, as seen in the following results.

\subsection{Displacement calibration}

For DC driving voltages, the speaker was found to have a displacement vs. voltage characteristic shown in Fig. 9. The displacement was essentially linear for nominal driving voltage $=-3.0$ to +3.0 volts, with a slope of approximately 0.36 . The error in displacement measurements $\partial d \approx 0.05 \mathrm{~mm}$. The displacement to displacement error ratio, $\partial d / d$ can dominate uncertainty in parameter estimation, and therefore must be minimised (Sec. 2). For accurate sample characterization, linearity is required to create a mechanical square wave (Fig. 7), and repeatability is required for high accuracy.

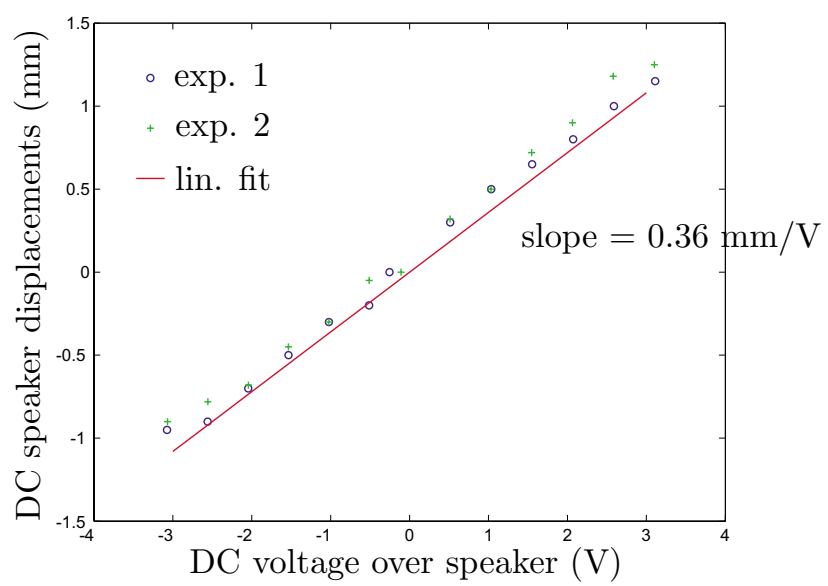

Figure 9. DC linearity of speaker displacement. DC linearity of the 4" RCA mid-range speaker. This data was determined manually using a micron-resolution manual stage to measure the displacement at each different DC bias voltage. The two measured plots give an indication of the repeatability of the speaker's motion. The linear plot gives an indication of the linearity of the speaker.

The DC characteristics of the speaker are less important than its AC, or dynamic, characteristics. The most important feature of the system's dynamic characteristics is that it accurately provides a square-wave motion, and therefore a $y_{d}$ (and $y_{m}$ ) is accurately detected. This accuracy can be estimated by comparing a normal TDS difference measurement, $\left(y_{r}-y_{s}\right) / 2$, to a DTDS measurement $y_{d}$. The accuracy is quantified by the spectral ratio of the two measurements over a certain frequency range where the T-ray signal is strong, for example 0.3 to $1.1 \mathrm{THz}$.

To experimentally determine the best modulation amplitude and frequency, measurements of $y_{s}, y_{r}$ and $y_{d}$ were taken with the T-ray spectrometer and a sample of anhydrous hexanes (approximately 10-mL in the HDPE bag).

Figure 10 shows the experimentally-measured accuracy of the DTDS measurements for increasing modulation frequency. The modulation frequency is limited to a range of approximately $2<f_{3}<10$. These measurements were all made at a modulation amplitude of $1 \mathrm{~V}$ AC. The ref line indicates a ratio of 1.6, which is expected between $\left(y_{r}-y_{s}\right) / 2$ and $y_{d}$. Using double modulation, we expect the amplitude of

$$
y_{d}=A \times\left(y_{r}-y_{s}\right)
$$

where $A=0.5 \times 0.6$. The factor of 0.5 arises from double modulation, and the factor of 0.6 accounts for the LIA measuring only the fundamental sine component of the actual square wave. These results indicate an maximum modulation frequency of about $10 \mathrm{~Hz}$ for this hardware.

The accuracy of DTDS measurements at different amplitudes was measured using the same sample of an organic solvent (an anhydrous mix of hexanes) and a 10-Hz modulation frequency. The displacement amplitude 


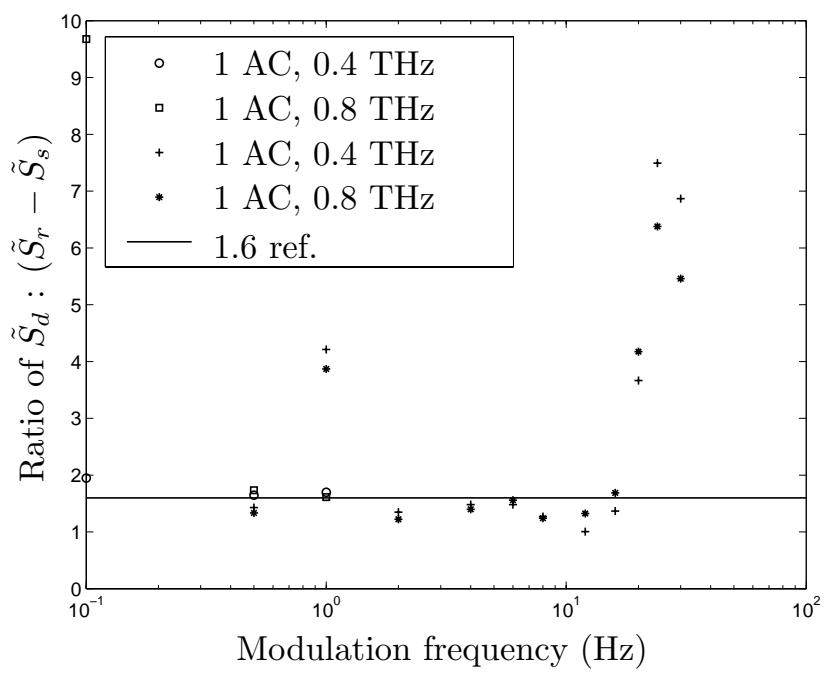

Figure 10. Optimal modulation frequency. How the accuracy of liquid DTDS decreases at high and low modulation frequencies. The ref line indicates a ratio 1.6, which means the DTDS measurement is $100 \%$ accurate. All measurements were made with a displacement amplitude of $1 \mathrm{~V}$ AC. The plots for 0.4 and $0.8 \mathrm{THz}$ are spectral components extracted from the pulsed waveforms. The plots for different T-ray frequencies have similar responses to the modulation frequency. This data was measured with a sample of anhydrous hexanes, chosen for its low T-ray absorption.

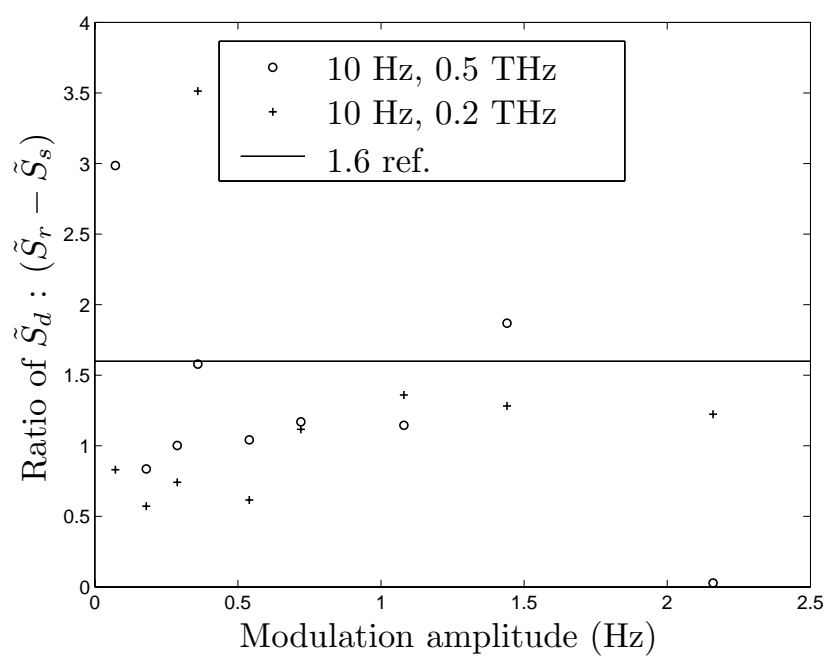

Figure 11. Optimal modulation amplitude. How the accuracy of liquid DTDS decreases at high and low modulation amplitudes. The Ref line indicates a ratio 1.6, which means the DTDS measurement is $100 \%$ accurate.

$d$ was limited to about $2.2 \mathrm{~mm}$. Figure 11 shows the highest accuracy of the DTDS measurements occurs at a displacement of $1.6 \mathrm{~mm}$.

The most accurate measurements are taken at $10 \mathrm{~Hz}$ with a displacement of $1.6 \mathrm{~mm}$. Figure 12 shows a direct comparison of the waveforms from DTDS $\left(y_{d}\right)$ and normal TDS. The large overlap of the waveforms experimentally verifies Eq. (18).

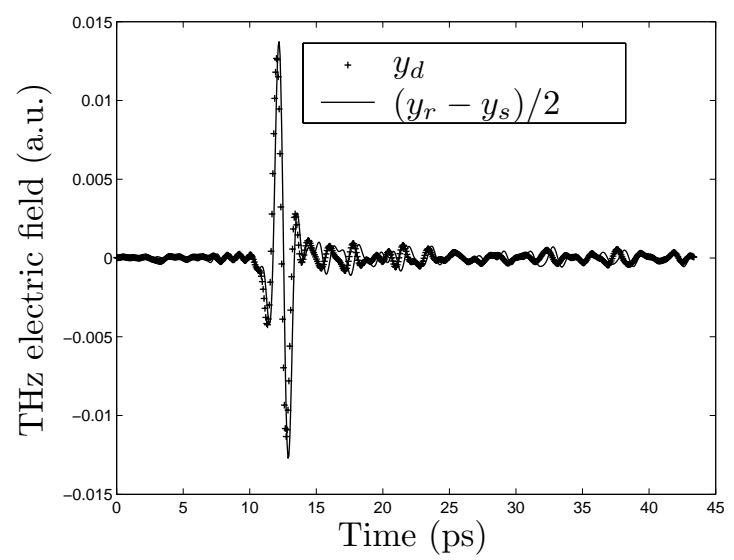

Figure 12. DTDS $y_{d}$ and $\left(y_{r}-y_{s}\right) / 2$. Comparison of $y_{d}$ and $\left(y_{r}-y_{s}\right) / 2$. The waveforms have been smoothed with a bandpass filter set at T-ray frequencies with highest SNR.

The biggest problem with this system was the inaccuracy in the displacement measurements. The error due to $\delta d$ is far greater than the error due to $\delta T$. For example, Table 1 shows approximate error levels for a typical 
Table 1. Error sources in liquid DTDS. Typically in a spectroscopy experiment, the following values are found for $0.5 \mathrm{THz}$. For repeatability of the T-ray spectrum, $\phi=22.30, \partial \phi=0.037, \rho=0.67$ and $\partial \rho=0.020$. For the thickness accuracy with $d=1.6 \mathrm{~mm}$, a manual estimate has $\partial d=5 \mu \mathrm{m}$ and a DTDS estimate has $\partial d=0.1 \mathrm{~mm}$, limited by the audio speaker. These results come from actual measurements of anhydrous dioxane. The error estimates are calculated using the equations in Sec. 2.2 .

\begin{tabular}{|c|c|c|c|}
\hline Source & Parameter & Error & $(d$ accuracy) \\
\hline due to $\partial T$ & $\partial n$ & 0.0044 & \\
& $\partial \kappa$ & 0.0025 & \\
\hline due to $\partial d$ & $\partial n$ & 0.0042 & (manual) \\
& & 0.083 & (DTDS) \\
& $\partial \kappa$ & 0.000075 & (manual) \\
& & 0.0015 & (DTDS) \\
\hline
\end{tabular}

spectroscopy measurements. The greatest source of error comes from inaccuracies in the measurement of $d$. The mechanical displacement accuracy can be greatly increased using a number of methods, include real-time external displacement measurement and closed-loop control. One method of achieving a very low $\partial d$ is to use a piezo translation system, as discussed in Sec. 5.1.

\subsection{Experimental examples of uncertainty}

To confirm the equations of Sec. 2.2, this section describes the experimental repeatability and estimated uncertainty in actual dual-thickness experiments. 6 T-ray TDS measurements of anhydrous dioxane were taken and the standard deviation was estimated using MATLAB. Then Eq. (15) was used to calculate the predicted uncertainty. The calculated uncertainty spectrum is shown in Figs. 13(a) and 13(b). The actual uncertainty in a series of measurements can be estimated from the standard deviation of a series of estimated values of $n$ and $\kappa$. As shown in Figs. 13(b) and 13(a), the equations provide an accurate estimate or an over-estimation of the error. These results confirm the accuracy of the equations used above to quantify the sources of uncertainty.

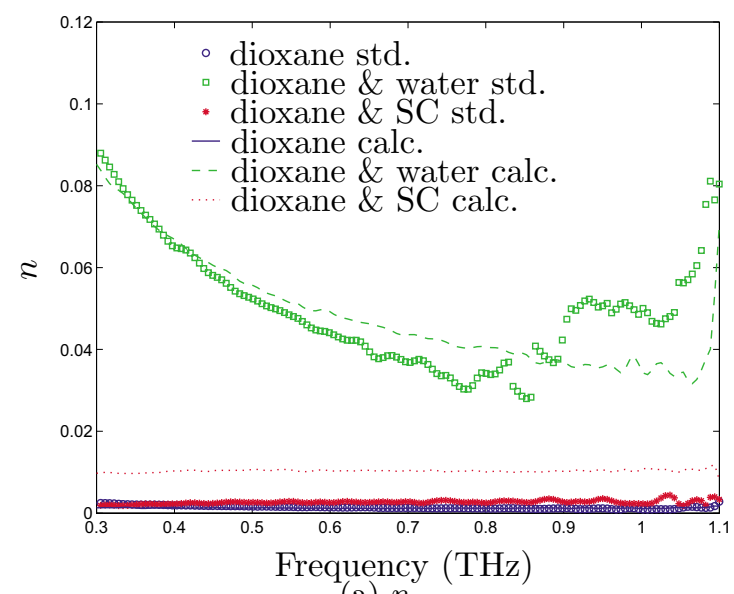

(a) $n$

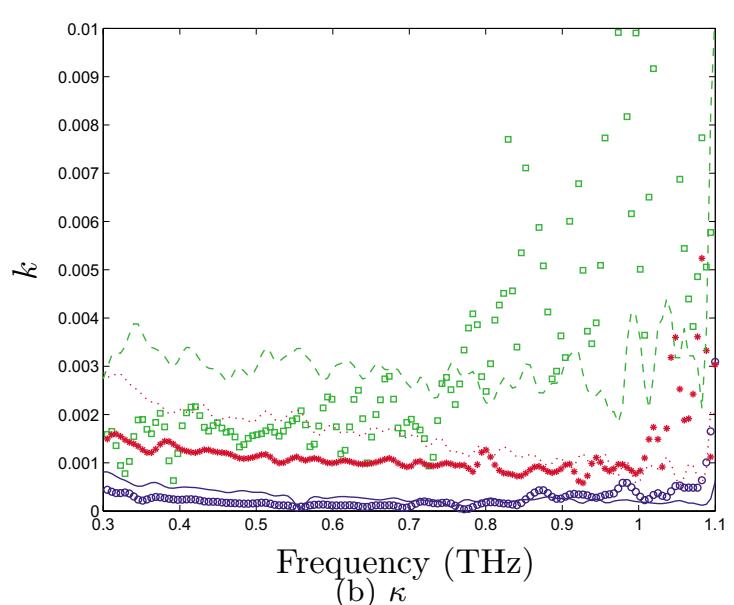

(b) $\kappa$

Figure 13. Experimental uncertainties. The uncertainties are estimated with MATLAB 's standard deviation function from six repeated experimental measurements. The points represent the standard deviation of the estimated values. The lines represent Eq. (15). The three samples are: (i) anhydrous dioxane, (ii) dioxane \& water, and (iii) dioxane \& a protein suspension (SC).

\section{CONCLUSION}

The results demonstrated a novel implementation of double-modulated DTDS for liquid spectroscopy. The prototype system confirmed a reduction in noise at an increased modulation frequency, and confirmed the 
accuracy of Eq. (15). The main limitation of the prototype was the dominant mechanical noise at higher modulation frequencies and amplitudes.

\subsection{Future work}

In future work the sample holder system will be implemented using a piezo-electric driven Teflon window. The advantage of a piezoelectric modulator is the extreme accuracy of movement, with $\partial d / d$ better than $10^{-4}$. A photograph of the proposed piezoelectric modulator is shown in

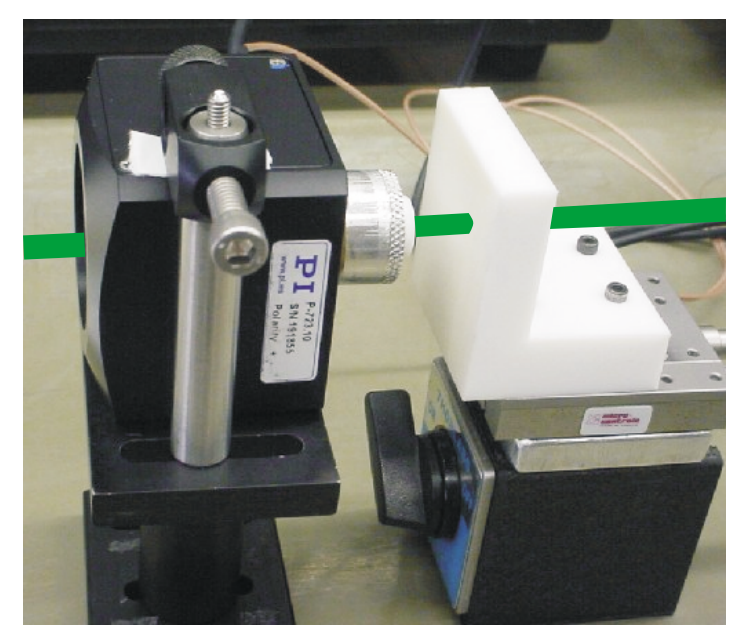

Figure 14. Piezoelectric sample modulator. The modulated Teflon window is driven by a piezoelectric microscope objective positioner manufactured by PI (Germany). The positioner has a travel of $350 \mu \mathrm{m}$, closed-loop resolution of $10 \mathrm{~nm}$ and a loaded resonant frequency of $100 \mathrm{~Hz}$. It will be used to characterise highly-absorbing liquids. The mechanical noise will be far less than that of the audio speaker modulator.

\section{SUMMARY}

The theoretical analysis in this paper, supported by preliminary experiments, indicates that rapid sample modulation can increase the accuracy of T-ray transmission spectroscopy of liquids by over 100 times. An increase in the accuracy of liquid parameter estimation by 2 or 3 orders of magnitude will be extremely valuable in many T-ray liquid experiments, including observing solvation processes and biomolecules in solution or suspension. The development of a piezo-driven DTDS stage will enable highly-accurate measurements of highly-absorbing liquids, which is a step towards studying biological systems with T-rays.

T-ray systems are not only used for spectroscopy, and one of the key developments of the last decade is extending T-rays into 2 and 3 dimensions, to T-ray imaging.

\section{ACKNOWLEDGMENTS}

We would like to acknowledge support from the U.S. National Science Foundation, the U.S. Army Research Office, the Australian Fulbright Commission and the Australian Research Council.

\section{REFERENCES}

1. S. P. Mickan, D. Abbott, J. Munch, X. C. Zhang, and T. van Doorn, "Analysis of system trade-offs for terahertz imaging," in Proceedings of SPIE - Electronics and Structures for MEMS, 3891, pp. 226-237, (Gold Coast, QLD, Australia), 1999.

2. S. P. Mickan, D. Abbott, J. Munch, X.-C. Zhang, and T. van Doorn, "Analysis of system trade-offs for terahertz imaging," Microelectronics Journal (Elsevier) 31(7), pp. 503-514, 2000. 
3. P. H. Siegel, "Terahertz technology," IEEE Transactions on Microwave Theory and Techniques 50(3), pp. 910-928, 2002.

4. S. P. Mickan and X.-C. Zhang, "T-ray sensing and imaging," in Terahertz Sensing Technology: Electronic Devices 83 Advanced Technology, D. Woolard, M. S. Shur, and W. Leorop, eds., Selected Topics in Electronics and Systems, ch. 8, Word Scientific Publishing Company, 2003. accepted 22 Oct 2002.

5. S. P. Mickan, J. S. Dordick, J. Munch, D. Abbott, and X.-C. Zhang, "Pulsed THz protein spectroscopy in organic solvents," in Conference on Lasers and Electro-Optics '02, p. 640, IEEE LEOS \& OSA, (Long Beach, CA, U.S.A.), 2002.

6. S. P. Mickan, J. S. Dordick, J. Munch, D. Abbott, and X.-C. Zhang, "Terahertz spectroscopy of bound water in nano suspensions," in Proceedings of SPIE - Biomedical Applications of Micro- and Nanoengineering, D. Nicolau, ed., 4937, pp. 49-61, Melbourne, Australia, 2002.

7. Z. Jiang, M. Li, and X.-C. Zhang, "Dielectric constant measurement of thin films by differential time-domain spectroscopy," Applied Physics Letters 76(22), pp. 3221-3223, 2000.

8. S. P. Mickan, K. S. Lee, T.-M. Lu, J. Munch, D. Abbott, and X.-C. Zhang, "Double modulated differential THz-TDS for thin film dielectric characterization," Microelectronics Journal (Elsevier) 33(12), pp. 1033$1042,2002$.

9. S. P. Mickan, D. Abbott, J. Munch, and X. C. Zhang, "Noise reduction in terahertz thin film measurements using a double modulated differential technique," Fluctuation and Noise Letters 2(1), pp. 13-29, 2002.

10. S. P. Mickan, A. Menikh, H. Liu, C. A. Mannella, D. Abbott, J. Munch, and X.-C. Zhang, "Label-free bioaffinity detection using terahertz technology," Physics in Medicine and Biology 47(21), pp. 3789-3796, 2002.

11. S. P. Mickan, A. Menikh, C. A. Mannella, D. Abbott, J. Munch, and X.-C. Zhang, "Amplification and modelling of bioaffinity detection with terahertz spectroscopy," in Proceedings of SPIE - Biomedical Applications of Micro- and Nanoengineering, D. Nicolau, ed., 4937, pp. 334-342, Melbourne, Australia, 2002.

12. L. Thrane, R. H. Jacobsen, P. U. Jepsen, and S. R. Keiding, "THz reflection spectroscopy of liquid water," Chemical Physics Letters 240, pp. 330-333, 1995.

13. C. Rønne, L. Thrane, P.-O. Åstrand, A. Wallqvist, K. V. Mikkelsen, and S. R. Keiding, "Investigation of the temperature dependence of dielectric relaxation in liquid water by $\mathrm{THz}$ reflection spectroscopy and molecular dynamics simulation," Journal of Chemical Physics 107(14), pp. 5319-5331, 1997.

14. C. Rønne and S. R. Keiding, "THz reflection spectroscopy of $\mathrm{H}_{2} \mathrm{O}(\mathrm{l})$ and $\mathrm{D}_{2} \mathrm{O}(\mathrm{l})$," in Ultrafast Phenomena XI, T. Elsaesser, J. G. Fujumoto, D. A. Wiersma, and W. Zinth, eds., Springer Series in Chemical Physics 63, pp. 568-570, Springer-Verlag, Berlin, 1998.

15. I. H. Libon, M. Hempel, S. Seitz, N. E. Hecker, J. Feldmann, A. Hayd, G. Zundela, D. Mittleman, and M. Koch, "THz spectroscopy of polar liquids," in Proceedings of SPIE - Terahertz Spectroscopy and Applications, 3617, pp. 24-29, (San Jose, CA, U.S.A.), 1999.

16. J. E. Pedersen and S. R. Keiding, "THz time-domain spectroscopy of nonpolar liquids," IEEE Journal of Quantum Electronics 28(10), pp. 2518-2522, 1992.

17. J. T. Kindt and C. A. Schmuttenmaer, "Far-infrared dielectric properties of polar liquids probed by femtosecond terahertz pulse spectroscopy," Journal of Physical Chemistry 100(24), pp. 10373-10379, 1996.

18. M. C. Beard, G. M. Turner, D. S. Venables, and C. A. Schmuttenmaer, "Two-dimensional time-resolved THz spectroscopy of solvent response to photoexcitation," in Trends in Optics and Photonics. Twelfth International Conference on Ultrafast Phenomena, 43, pp. 592-594, OSA, (Charleston S.C. USA), 2000.

19. M. C. Beard and C. A. Schmuttenmaer, "Using the finite-difference time-domain pulse propagation method to simulate time-resolved THz experiments," Journal of Chemical Physics 114(7), pp. 2903-2909, 2001.

20. D. S. Venables, A. Chiu, and C. A. Schmuttenmaer, "Structure and dynamics of nonaqueous mixtures of dipolar liquids. I. Infrared and far-infrared spectroscopy," Journal of Chemical Physics 113(8), pp. 3243$3248,2000$.

21. D. S. Venables and C. A. Schmuttenmaer, "Structure and dynamics of nonaqueous mixtures of dipolar liquids. II. Molecular dynamics simulations," Journal of Chemical Physics 113(8), pp. 3249-3260, 2000.

22. D. S. Venables and C. A. Schmuttenmaer, "Spectroscopy and dynamics of mixtures of water with acetone, acetonitrile, and methanol," Journal of Chemical Physics 113(24), pp. 11222-11236, 2000. 
23. D. M. Mittleman, J. Cunningham, M. C. Nuss, and M. Geva, "Noncontact semiconductor wafer characterization with the terahertz Hall effect," Applied Physics Letters 71(1), pp. 16-18, 1997.

24. M. L. T. Asaki, A. Redondo, T. A. Zawodzinski, and A. J. Taylor, "Dielectric relaxation of electrolyte solutions using terahertz transmission spectroscopy," Journal of Chemical Physics 116(19), pp. 8469-8482, 2002.

25. E. Zoidis, M. Besnard, and J. Yarwood, "Far infrared spectroscopic studies of the molecular dynamics and interactions of pyridine in organic solvents," Chemical Physics Letters 203, pp. 233-243, 1996.

26. C. Rønne, K. Jensby, B. J. Loughnane, J. Fourkas, O. F. Nielsen, and S. R. Keiding, "Temperature dependence of the dielectric function of $\mathrm{C}_{6} \mathrm{H}_{6}(\mathrm{l})$ and $\mathrm{C}_{6} \mathrm{H}_{5} \mathrm{CH}_{3}(\mathrm{l})$ measured with $\mathrm{THz}$ spectroscopy," Journal of Chemical Physics 113(9), pp. 3749-3756, 2000.

27. L. Duvillaret, F. Garet, and J.-L. Coutaz, "A reliable method for extraction of material parameters in terahertz time-domain spectroscopy," IEEE Journal of Selected Topics in Quantum Electronics 2(3), pp. 739746, 1996.

28. S. P. Mickan and X.-C. Zhang, "T-ray sensing and imaging," International Journal of High Speed Electronics and Systems , 2003. accepted 22 Oct 2002.

29. M. Born and E. Wolf, Principles of Optics, Cambridge University Press, 6th ed., 1997.

30. G. Arjavalingam, Y. Pastol, J.-M. Halbout, and G. V. Kopcsay, "Broad-band microwave measurements with transient radiation from optoelectronically pulsed antennas," IEEE Transactions on Microwave Theory and Techniques 38(5), pp. 615-621, 1990.

31. L. Duvillaret, F. Garet, and J. L. Coutaz, "Influence of noise on the characterization of materials by terahertz time-domain spectroscopy," Journal of the Optical Society of America B: Optical Physics 17(3), pp. 452-461, 2000 .

32. S. P. Mickan, K.-S. Lee, T.-M. Lu, E. Barnat, J. Munch, D. Abbott, and X.-C. Zhang, "Thin film characterization using terahertz differential time-domain spectroscopy and double modulation," in Proceedings of SPIE - Electronics and Structures for MEMS II, N. W. Bergmann, ed., 4591, pp. 197-209, (Adelaide, Australia), 2001. 